\title{
Awareness, attitude and usage of probiotics for the prevention of antibiotic-associated diarrhoea among caregivers and HCPs in Poland: a cross-sectional study
}

\author{
Michał F. Wronowski ${ }^{1,2}$, Maria Kotowska' ${ }^{1}$ Monika Wanke², Artur Kotowski ${ }^{3}$, Jan Bukowski ${ }^{1}$, \\ Piotr Albrecht ${ }^{1}$ \\ 'Department of Pediatric Gastroenterology and Nutrition, Medical University of Warsaw, Poland \\ 2Department of Pediatrics with Clinical Assessment Unit, Medical University of Warsaw, Poland \\ ${ }^{3}$ General Association for Good Clinical Practice, Warsaw, Poland
}

\section{ABSTRACT}

Objectives: This cross-sectional study aimed at obtaining and analyzing data on awareness, attitude and usage of probiotics for AAD prevention in children among caregivers and doctors in Poland.

Material and methods: This was a self-administered survey of the children's caregivers. The survey evaluated caregivers' opinion on probiotics efficacy in AAD prevention, obtained data about probiotic preparations used and their source of recommendation. Probiotics were classified basing on ESPGHAN recommendations in AAD prevention.

Results: Probiotics were used in 99.4\% $(n=456 / 463) .190$ patients used recommended strains, 162 patients used preparations without proven efficacy. In $87 \%$ of cases $(n=397 / 456)$, probiotics were advised by doctors, out of which $56 \%$ were recommended strains. $32 \%$ of them had proven efficacy.

Conclusions: Our study showed a positive attitude of caregivers towards probiotics for AAD prevention. However, almost half of the preparations were without proven efficacy. HCPs education on the field of probiotics is warranted.

KEY WORDS:

children, antibiotic-associated diarrhoea, probiotics, Clostridioides difficile.

\section{INTRODUCTION}

According to $\mathrm{FAO} / \mathrm{WHO}$ definition, probiotics are live microorganisms that confer a health effect on the host when consumed in adequate amounts [1]. Probiotics are used for treatment or prevention on various indications. Only a few well-established effects include antibiotic-associated diarrhoea prevention, acute gastroenteritis, general gut health improvement, treatment of obesity, allergy and atopic diseases in children, hypercholesterolemia, cancer prevention and hepatic encephalopathy [2]. Probiotics effects are strain and dose-dependent $[3,4]$. Saccha- romyces boulardi and Lactobacillus rhamnosus LGG are effective and recommended in reducing AAD incidence (risk reduction, RR 0.43-0.48) [5, 6].

To our knowledge, there have been no studies assessing parents' knowledge, attitudes and usage of probiotics usage in the prevention of antibiotic-associated diarrhoea in children.

There are several dozen probiotic preparations available on the Polish market. A large proportion of them contains strains of microorganisms with unproven activity preventing the development of AAD. Thus, depending on the indication, healthcare personnel (HCP) should

\section{ADDRESS FOR CORRESPONDENCE:}

Michał F. Wronowski, Department of Pediatric Gastroenterology and Nutrition, Medical University of Warsaw,

Poland, e-mail: mwronowski@wum.edu.pl 
recommend preparation containing specific strains however, the choice is usually left to the childcarer [7].

We conducted this study to contribute a better knowledge of probiotics usage in AAD prevention in children.

\section{MATERIAL AND METHODS}

This was a cross-sectional study contributing to improve knowledge on probiotics in the prevention of AAD in children. The design of the study was preceded by a literature review. The pilot to asses content and face validity of the questionnaire was conducted on 15 childcarers of hospitalized children. We spread the self-administered anonymous survey consisting of 6 questions (Table 1) among random caregivers of children hospitalized or outpatient at the Medical University of Warsaw Public Pediatric Teaching Hospital. The inclusion criterium was antibiotic therapy within a year before excluding current hospitalization. We enrolled 463 individuals in the study. Childcarers fulfilled the questionnaire with no help from the study staff; after completion, they put questionnaires into the closed ballot box. The participants were informed and assured of anonymity and confidentiality - we obtained no personal data. Based on the data collected, it is not possible to identify the survey participants.

This study aimed to determine as following:

- Whether probiotics are used in the AAD prevention if so, tradename was obtained?

- Did child use preparations with proven effectiveness in $\mathrm{AAD}$ risk reduction?
- Do childcarers believe that the use of probiotics reduces the risk of AAD development?

- Did parents or legal guardians who believe that the use of probiotics can be effective in AAD risk reduction used a preparation with proven effectiveness?

- Who recommended the usage of specific preparation?

- Did physicians and pharmacists recommend the use of a product with proven effectiveness in the AAD risk reduction?

We defined the preparation of proven effectiveness in the AAD risk reduction (Table 2) as a preparation containing probiotic strain recommended by ESPGHAN to prevent AAD - Lactobacillus rhamnosus LGG Saccharomyces boulardii at the dose of $\geq 5$ billion CFU $[8,9]$. A doctor, a pharmacy employee or a person not related to healthcare were to indicate as a source of recommendation of probiotic preparation.

After enrollment was completed, we listed the tradename of preparations that were indicated by parents and assigned them to one of two groups:

- Preparations with proven effectiveness in reducing the risk of AAD: a medicine or non-medicinal product containing a probiotic strain at the dose $\geq 5$ billion CFU recommended by the manufacturer in this indication.

- Preparations with no or insufficient data on AAD risk reduction: a medicine or non-medicinal product containing a probiotic strain with a proven antibiotic risk reduction for $\mathrm{AAD}$ at the dose of $\leq$ than 5 billion $\mathrm{CFU}$

TABLE 1. Self-administered questionnaire

Please complete this questionnaire to assess the use of probiotics in the prevention of post-antibiotic diarrhoea in children. The questionnaire is anonymous. We will use its results to determine caregivers' attitudes towards probiotics in the prevention of antibiotic-associated diarrhoea. The results of this study will contribute to improving the effectiveness of probiotics use.

1. Has your child been treated with an antibiotic within the last year? $\square$ YES $\square$ NO

2. Did you use any prevention of antibiotic-associated diarrhoea (AAD) during antibiotic treatment? $\square$ YES $\square$ NO

3. In your opinion, prevention methods may reduce the risk for AAD development? $\square$ YES $\square$ NO

4. Please indicate the prevention product your child took during the antibiotic therapy:

$\square$ Probiotic product $\quad \square$ kefir $\quad \square$ yoghurt
$\square$ Other:
$\square$ A combination of the above:

5. Please indicate who recommended such a method of $A A D$ prevention: $\square$ Medical doctor $\square$ Pharmacy employer $\quad \square$ Person unrelated to medicine

6. If a probiotic product was administered, please provide its name:

$\square$ Name of the preparation:

$\square$ I don't remember the name of the product. 
TABLE 2. Classification of probiotic products. 1: Preparation with proven effectiveness in reducing the risk of AAD - medicine; 2: Non - medicinal preparation with proven effectiveness in reducing the risk of $A A D ; 3$ : Preparations with no or insufficient data on AAD risk reduction

\begin{tabular}{|c|c|c|c|}
\hline No. & Dose & Probiotic strains & Classification* \\
\hline 1. & $4 \times 10^{9} \mathrm{CFU}$ in total & $\begin{array}{l}\text { Lactobacillus acidophilus, Bifidobacterium bifidum, Lactobacillus rhamnosus, Strepto- } \\
\text { coccus thermophilus }\end{array}$ & 3 \\
\hline 2. & $5 \times 10^{9} \mathrm{CFU}$ & Lactobacillus rhamnosus GG ATCC 53103 & 2 \\
\hline 3. & $4 \times 10^{9} \mathrm{CFU}$ in total & Bifidobacterium lactis BS01, Lactobacillus acidophilus LA02 & 3 \\
\hline 4. & $6 \times 10^{9} \mathrm{CFU}$ in total & Lactobacillus rhamnosus GG ATCC 53103, Lactobacillus reuteri, Lactobacillus gasseri & 3 \\
\hline 5. & $4 \times 10^{9} \mathrm{CFU}$ in total & $\begin{array}{l}\text { Lactobacillus acidophilus, Bifidobacterium bifidum, Lactobacillus rhamnosus, } \\
\text { Streptococcus thermophilus }\end{array}$ & 3 \\
\hline 6. & $0,1 \times 10^{9} \mathrm{CFU}$ & L. reuteri DSM 17938 & 3 \\
\hline 7. & $0,4 \times 10^{9} \mathrm{mld}$ CFU & Lactobacillus casei DN-114 001 & 3 \\
\hline 8. & $6 \times 10^{9} \mathrm{mld}$ CFU & Lactobacillus rhamnosus GG ATCC 53103) & 2 \\
\hline 9. & $5 \times 10^{9} \mathrm{mld}$ CFU & Saccharomyces boulardii B01 & 2 \\
\hline 10. & $5 \times 10^{9} \mathrm{CFU}$ & Saccharomyces boulardii CNCM I-745 & 1 \\
\hline 11. & $6 \times 10^{9} \mathrm{CFU}$ & Lactobacillus rhamnosus GG ATCC 53103 & 2 \\
\hline 12. & $3 \times 10^{9} \mathrm{CFU}$ in total & $\begin{array}{l}\text { Lactobacillus acidophilus Rosell-52, Bifidobacterium infantis Rosell-33, } \\
\text { Bifidobacterium bifidum Rosell-71 }\end{array}$ & 3 \\
\hline 13. & $2 \times 10^{9} \mathrm{CFU}$ in total & Lactobacillus rhamnosus R0011, Lactobacillus helveticus R0052 & 3 \\
\hline 14. & $10 \times 10^{9} \mathrm{CFU}$ in total & $\begin{array}{l}\text { Lactobacillus plantarum PL 02, Lactobacillus rhamnosus KL 53A, Bifidobacterium } \\
\text { longum PL } 03\end{array}$ & 3 \\
\hline 15. & $2 \times 10^{9} \mathrm{CFU}$ in total & $\begin{array}{l}\text { Lactobacillus rhamnosus Pen, Lactobacillus rhamnosus E/N; Lactobacillus rhamnosus } \\
\text { 0xy }\end{array}$ & 3 \\
\hline 16. & $2 \times 10^{9}$ CFU in total & Lactobacillus acidophilus LA-5, Bifidobacterium animalis lactis BB-12 & 3 \\
\hline 17. & $4,5 \times 10^{9}$ CFU in total & $\begin{array}{l}\text { Lactobacillus helveticus SP 27, Bifidobacterium longum BI-05, Lactobacillus plantarum Lp-115, } \\
\text { Lactobacillus casei Lc-11, Lactococcus lactis LI-23, Lactobacillus rhamnosus Lr-32, } \\
\text { Bifidobacterium bifidum Bb-02, Streptococcus thermophilus St-21, Bifidobacterium breve Bb-03 }\end{array}$ & 3 \\
\hline 18. & $4 \times 10^{9} \mathrm{CFU}$ in total & Saccharomyces bouldarii DBVPG 6763, Lactobacillus rhamnosus GG ATCC 53103 & 3 \\
\hline 19. & $10 \times 10^{9} \mathrm{CFU}$ & Lactobacillus plantarum 299 & 3 \\
\hline 20. & $1,5 \times 10^{9} \mathrm{CFU}$ in total & $\begin{array}{l}\text { Lactobacillus acidophilus La-5, Lactobacillus Brodecki subs. Bulgaricus Lb-Y27, } \\
\text { Bifidobacterium lactis Bb-12 }\end{array}$ & 3 \\
\hline 21. & $450 \times 10^{9} \mathrm{CFU}$ in total & $\begin{array}{l}\text { Streptococcus thermophilus DSM24731, Bifidobacterium Iongum DSM24736, } \\
\text { Bifidobacterium breve DSM24732, Bifidobacterium infantis DSM24737, Lactobacillus } \\
\text { acidophilus DSM24735, Lactobacillus plantarum DSM24730, Lactobacillus paracasei } \\
\text { DSM24733, Lactobacillus delbrueckii ssp. bulgaricus DSM24734 }\end{array}$ & 3 \\
\hline 22. & $4,08 \times 10^{9} \mathrm{CFU}$ in total & $\begin{array}{l}\text { Bifidobacterium lactis BS01, Lactobacillus acidophilus LA02, Streptococcus thermo- } \\
\text { philus FP4, Lactobacillus delbrueckii subsp. bulgaricus LDB01, Lactobacillus casei LC03, } \\
\text { Saccharomyces boulardii CNCM I-3799 } 299\end{array}$ & 3 \\
\hline 23. & $5 \times 10^{9} \mathrm{CFU}$ & Saccharomyces boulardii CNCM-I-1079 & 2 \\
\hline
\end{tabular}

$A A D$ - antibiotic-associated diarrhoea; CFU - colony-forming unit

recommended by the manufacturer in this indication or preparation containing probiotic strain with no proven effect in AAD risk reduction or in combination with yoghurt/kefir.

\section{RESULTS}

We obtained 463 questionnaires filled in by childcarers. 456 (98.48\%) children received probiotic preparation during antibiotic therapy; 23 different probiotic preparations were used. The majority of caregivers
( $n=378 / 463 ; 81.64 \%)$ believed that probiotics are effective in AAD prevention, the minority was of the opposite opinion ( $n=28 / 463 ; 6.47 \%)$, some did not express an opinion on that matter ( $n: 56 / 463 ; 12,09 \%)$. In 34 cases $(7.34 \%)$, patients received medicines of proven effectiveness in the AAD risk reduction, 156 (33.69\%) used non-medicinal products of proven effectiveness, 162 (34.98\%) patients used preparations with no or insufficient data to recommend (Table 3). 104 (22.4\%) caregivers could not recall the trade name of the product used, 7 did not use probiotic products. 
TABLE 3. Categorization of probiotic preparations - summary

\begin{tabular}{|c|c|}
\hline & $\mathrm{N}(\%)$ \\
\hline \multicolumn{2}{|l|}{ Preparation effective in AAD risk reduction } \\
\hline Medicine & $34(7.4 \%)$ \\
\hline Non-medicinal & $156(34.2 \%)$ \\
\hline $\begin{array}{l}\text { Preparation with no proven effectiveness on AAD } \\
\text { risk reduction }\end{array}$ & $162(35,5 \%)$ \\
\hline $\begin{array}{l}\text { No possibility of determining the preparation } \\
\text { name }\end{array}$ & $104(22,8 \%)$ \\
\hline
\end{tabular}

$A A D$ - antibiotic-associated diarrhoea

In $87 \%(n=397 / 456)$ of cases, probiotics preparations were advised by doctors, out of which $56 \%$ were products of proven effectiveness. $5.5 \%$ of products $(n=25 / 456)$ were based on pharmacy employee recommendation, however only $32 \%$ of proven efficacy. Surprisingly, individuals unrelated to medicine $(7.5 \%)$ recommended products of proven efficacy in $48 \%$ of cases.

$378(82,89 \%)$ responders believed that probiotics might reduce the risk of the AAD. In this group, we analyzed the data from respondents who remembered the name of the preparation. 250 patients used preparations recommended by physicians, among them $54,8 \%$ were with proven effectiveness $(n=137 / 250)$; pharmacy employee recommended probiotics with proven efficacy in $23,5 \%(n=4 / 17)$.

\section{DISCUSSION}

To our knowledge, this is the first cross-sectional study assessing parental attitudes towards probiotics in correlation with their usage in AAD prevention.

The study revealed that a general childcarer's perspective towards probiotics in AAD prevention in children is positive. In previous research, the approach to probiotics has been investigated in other areas. The group of childcarers' perspectives toward probiotics in other indications was generally positive, differing significantly from adults' attitudes [10].

Childcarers expect probiotic administration to prevent AAD effectively. They expect a risk reduction (RR) of at least 39\% [11]. This need may be met if probiotics recommended by ESPGHAN on this indication were used.

In our study, only $32 \%$ of pharmacists and $56 \%$ of doctors recommended probiotics aligning with ESPGHAN guidance on AAD prevention. It was associated with an increased risk of ineffective prevention of AAD, which may reduce cost-effectiveness and patients to become non-compliant to antibiotic treatment $[11,12]$.

Surprisingly, recommendations to use probiotics with non-proven RR reduction in AAD are made by paediatricians - the group of doctors declaring probiotics have a place in clinical practice and accepting the evidence behind their use in AAD prevention. At the same time, they point to the need for education on this subject. However,
$75 \%$ of HCP in the USA claimed not to read any recent peer-reviewed papers on probiotics [13].

Recommendation of preparations with no or insufficient data on AAD risk reduction by pharmacists that was shown in this study may be multifactorial. This group evaluated their knowledge on the field of probiotics with a median grade of 4 (good knowledge) on the 5-point Likert scale [14]. However, consideration should be made that pharmacists are generally not involved in OTC pharmacy purchases. There is the pressure exerted by pharmacy owners for high-profit economic efficiency. Thus the choice of OTC purchases might be influenced by the economy $[15,16]$.

An aspect of being considered independently is the registration status of the probiotic product. Depending on the country, registration procedures differ between medicines and non-medicinal products such as supplements. In European countries, Canada and USA supplements mostly require notification to a regulatory agency only, whilst medicinal products undergo the regulatory process as a drug $[17,18]$. Generally, products registered as medicines are considered to be of better quality. Several studies were conducted to assess the probiotics label's information conformity, showing that some of the products did not contain declared bacterial strains [19], product bacteria were not vial, colony form unit did not exceed the value from the label or contained toxin-producing bacteria [20]. Thus ESPGHAN released a call to enhance probiotic market control by the respective authorities and implement the standardization and validation control performed by reference laboratories to improve probiotic products quality [21].

Basing on the respondents' answers, we indirectly explored HCPs' prescribing attitudes on probiotics used for AAD prevention. Almost half of doctors recommendations were preparations with no proven effect on AAD prevention. This is inconsistent with doctors high self-assessment on their knowledge on this field. At the same time, HCP indicates the need for further education on the subject [22].

Another strength of the study is the large number of respondents. However, our study has limitations that may affect the generalizability of the results to the whole pediatric population. We conducted the survey exclusively on legal guardians of children presenting to the tertiary university hospital in Poland's capital. Thus, the responses obtained in the questionnaire may differ from those from smaller centres. Additionally, the self-administered survey was handed out and received by a researcher, which could have affected responders answers. Other limitation includes lack of social characteristics of respondents.

To conclude, this cross-sectional study of 463 individuals indicates childcarers believe probiotics can prevent AAD. This results in high usage during antibiotic therapy. Parents usually follow HCP recommendations on the selection of preparation. 
However, a significant number of HCP recommended probiotics with no proven effectiveness on AAD risk reduction. It may be associated with the ineffectiveness of AAD prevention.

In conclusion education of HCPs in the area of probiotics in prevention of AAD is warranted - this may increase the likelihood of its effectiveness. Our study showed a positive attitude of caregivers towards probiotics for AAD prevention. However, almost half of the preparations (in the majority advised by doctors) were without proven efficacy. In case of AAD occurrence, lack of effective prevention might lead to the loss of trust in probiotics effectiveness and unjustifiably increase AAD prevention's cost with no certainty of effectiveness.

\section{DISCLOSURE}

The authors declare no conflict of interest.

\section{REFERENCES}

1. Araya M, Morelli L, Reid G, et al. Guidelines for the evaluation of probiotics in food. Joint FAO/WHO Working Group Report on Drafting Guidelines for the Evaluation of Probiotics in Food 2002; 1-11.

2. Vyas U, Ranganathan N. Probiotics, prebiotics, and synbiotics: gut and beyond. Gastroenterol Res Pract 2012; 2012: 872716.

3. Guarino A, Lo Vecchio A, Canani RB. Probiotics as prevention and treatment for diarrhoea. Curr Opin Gastroenterol 2009; 25: 18-23.

4. Guarino A, Ashkenazi S, Gendrel D, et al. European Society for Pediatric Gastroenterology, Hepatology, and Nutrition/European Society for Pediatric Infectious Diseases evidence-based guidelines for the management of acute gastroenteritis in children in Europe: update 2014. J Pediatr Gastroenterol Nutr 2014; 59: 132-152.

5. McFarland L. Meta-Analysis of Probiotics for the Prevention of Antibiotic Associated Diarrhoea and the Treatment of Clostridium difficile Disease. Am J Gastroenterol 2006; 101: 812-822.

6. Goldenberg JZ, Lytvyn L, Steurich J, et al. Probiotics for the prevention of pediatric antibiotic-associated diarrhoea. Cochrane $\mathrm{Da}$ tabase of Syst Rev 2015; (12): CD004827.

7. Draper K, Ley C, Parsonnet J. A survey of probiotic use practices among patients at a tertiary medical centre. Benef Microbes 2017 8: 345-351.

8. Szajewska H, Canani RB, Guarino A, et al. Probiotics for the Prevention of Antibiotic-Associated Diarrhoea in Children. J Pediatr Gastroenterol Nutr 2016; 62: 495-506.

9. Guo Q, Goldenberg JZ, Humphrey C, et al. Probiotics for the prevention of pediatric antibiotic-associated diarrhoea. Cochrane $\mathrm{Da}$ tabase Syst Rev 2019; 4: CD004827.

10. Rijkers GT, Bimmel D, Grevers D, et al. Consumer perception of beneficial effects of probiotics for human health. Benef Microbes 2013; 4: 117-121.

11. Khanpour Ardestani S, Robinson JL, et al. Surveys of parents and clinicians concerning the minimally important difference of probiotic therapy for prevention of paediatric antibiotic-associated diarrhoea. BMJ Open 2019; 9: e024651.

12. Varughese CA, Vakil NH, Phillips KM. Antibiotic-associated diarrhoea: a refresher on causes and possible prevention with probiotics continuing education article. J Pharm Pract 2013; 26: 476-482.

13. Oliver L, Rasmussen H, Gregoire M, et al. Health Care Provider's Knowledge, Perceptions, and Use of Probiotics and Prebiotics. Topics in Clinical Nutrition 2014; 29: 139-149.
14. Fijan S, Frauwallner A, Varga L, et al. Health Professionals' Knowledge of Probiotics: An International Survey. Int J Environ Res Public Health 2019; 16: 3128

15. Kotecki JE. Factors Related to Pharmacists' Over-the-Counter Recommendations. J Community Health 2002; 27: 291-306.

16. Kennedy E, Moody M. An investigation of the factors affecting community pharmacists' selection of over the counter preparations. Pharm World Sci 2000; 22: 47-52.

17. Venugopalan V, Shriner KA, Wong-Beringer A. Regulatory oversight and safety of probiotic use. Emerg Infect Dis 2010; 16: 16611665.

18. Sanco D, Commission E. The use of substances with nutritional or physiological effect other than vitamins and minerals in food supplements study.

19. Szajewska H, Fordymacka A, Bardowski J, et al. Microbiological and genetic analysis of probiotic products licensed for medicinal purposes. Med Sci Monit 2004; 10: Br346-Br350.

20. Aureli P, Fiore A, Scalfaro C, et al. National survey outcomes on commercial probiotic food supplements in Italy. Int J Food Microbiol 2010; 137: 265-273.

21. Kolaček S, Hojsak I, Berni Canani R, et al. Commercial Probiotic Products: A Call for Improved Quality Control. A Position Paper by the ESPGHAN Working Group for Probiotics and Prebiotics. J Pediatr Gastroenterol Nutr 2017; 65: 117-124.

22. Wilson Z, Whitehead K. A cross sectional survey to assess healthcare professionals' attitudes to and understanding of probiotics. Clin Nutr ESPEN 2019; 34: 104-109. 